patients remain the most powerful tool for achieving greater patient satisfaction.

Reaside Clinic

Rubery, Rednal

Birmingham B45 9B

County Hospital

Hereford HRI 2ER

\section{Are psychiatric case-notes offensive?}

\section{DEAR SIRS}

The inclusion of truly offensive material in the case-notes of any patient has always been clearly unacceptable, regardless of whether or not patients are entitled in law to see their records. In the methods section of their study of case-notes from Charing Cross Hospital, Crichton et al (Psychiatric Bulletin, 1992), 16, 675-677) give a defining example of what they would rate as "extremely offensive". This was the comment: "a most unpleasant man". No doubt this could be taken as offensive, even extremely so by some readers, and such comments appeared in $62 \%$ of the case-notes reviewed. A most disturbing finding? We think not. On examining the appendix which contains examples of comments considered extremely offensive by all four raters, the degree of offensiveness of none of them struck us as coming up to that of "a most unpleasant man". To test this, we presented the comments in the appendix to an intelligent layperson (Margaret, our Section secretary) and asked her to rate them for offensiveness. She regarded comments (4) ("My greatest fear is that A. was unwell when she decided to marry this individual") and (9) ("He is a pitiful and lonely man") as "offensive", but thought none of them qualified for an "extremely offensive". The study authors have a valid point when they urge those writing in casenotes to avoid unfortunate and insensitive valueladen comments and gratuitous rudeness. Contrary to the conclusions of this study, most psychiatrists do not write offensive comments in case-notes. We believe that the authors have misled themselves through the downright silliness of what they appear to have deemed as offensive. Perhaps the greatest offence we can occasion our patients is to patronise them with robotic, politically correct language when we speak to them and write in their case-notes.

ROBERT HOWARD

SIMON LOVESTONE

Institute of Psychiatry

Denmark Hill

London SES 8 AF

DEAR SIRS

I read with Crichton et al's audit of psychiatric casenotes (Psychiatric Bulletin, 1992, 16,657-677). Their raters universally found such terms as 'a schizophrenic' and 'a depressive' extremely offensive because they claim these terms are stigmatising. Turning to the same month's edition of your sister journal (British Journal of Psychiatry) I noticed it too carried references to 'schizophrenics', 'bulimics' and 'heroin addicts'. By these criteria both the editors and contributors to the yellow journal are 'extremely offensive'.

The more serious point Crichton et al's paper raised is that whereas this usage is stigmatising in mental illnesses, it is considered neutral in physical illnesses; thus labelling someone 'a diabetic' is inoffensive. It seems clear that in an attempt to sound politically correct psychiatrists run the risk of colluding with the prejudice suffered by their patients elsewhere.

\section{The Maudsley Hospital}

MATTHEW HoTOPF

\section{Denmark Hill \\ London SE5 8 AZ}

\section{DeAR Sirs}

It is hardly surprising that psychiatric case-notes contain material which patients find offensive. It is in the nature of mental illness to be seen as stigmatising, so merely being a "case" inevitably causes offence to some people. This is particularly likely to be the case with psychiatric case-notes as our observations tend to be of a far more personal nature than those found in medical case-notes.

I would not disagree with advice on the need to be cautious in making entries into notes. However, I think we need to be more discriminating than the authors of this article suggest. There is an important distinction between those remarks which are gratuitously offensive, and those that offend but which are nevertheless true and may be important. We must guard against the trend for case-notes to be become increasingly bland and convey no useful information whatsoever. This does necessitate making entries which will cause offence, for example comments on somebody's dress, hygiene and appearance. It would cause offence to many people to read a comment that they smelt or looked peculiar, but these observations may be as important as noting the presence of hallucinations. It may be of crucial importance to know that somebody has a "tendency to become seriously disturbed" even if that person does not like to see it in his or her notes.

I believe the simplest rule is that no relevant fact should be omitted, however offensive it may seem, but that personal opinion should be limited to that which is clinically justifiable.

I. R. MEDLEY

North-East/Arnold Sector

Mental Health Team

Mandala Centre

Gregory Boulevard

Nottingham NG7 6LB 